\title{
Nuclear Magnetic Resonance Spectroscopy in the Detection and Characterisation of Cardiovascular Disease: Key Studies
}

\author{
Warren Yabsley, ${ }^{1}$ Shervanthi Homer-Vanniasinkam, ${ }^{2}$ and Julie Fisher ${ }^{1}$ \\ ${ }^{1}$ School of Chemistry, University of Leeds, Leeds LS2 9JT, UK \\ ${ }^{2}$ Leeds Vascular Institute, Leeds General Infirmary, Leeds LS1 3EX, UK \\ Correspondence should be addressed to Julie Fisher, j.fisher@chem.leeds.ac.uk
}

Received 9 August 2012; Accepted 29 August 2012

Academic Editors: K. Abe and Z. Yang

Copyright ( 2012 Warren Yabsley et al. This is an open access article distributed under the Creative Commons Attribution License, which permits unrestricted use, distribution, and reproduction in any medium, provided the original work is properly cited.

\begin{abstract}
Purpose. To review the available literature on the applications of nuclear magnetic resonance (NMR) spectroscopy in cardiovascular disease identification and aetiology. Methods. A systematic literature search of peer-reviewed journals for the period January 2000 to June 2012 was performed using EMBASE and Web of Knowledge (WOK) databases. Several hundred reports were identified. Abstract searching led to the selection of articles included. Results. Numerous studies show the breadth of applications of NMR spectroscopy in relation to cardiovascular disease including lipid and lipoprotein formation, structure, and drug interaction. Lipoprotein and metabolite profiles have been shown to vary with many manifestations of cardiovascular disease. Conclusion. The inherent accuracy and reproducibility of NMR spectroscopy allow subtle differences in lipoprotein and metabolic profiles to be detected. As more information is obtained and cardiovascular disease applications of NMR spectroscopy increase, there is potential that a route towards reducing the burden of this worldwide problem may become evident.
\end{abstract}

\section{Introduction}

The focus of this paper is cardiovascular disease, the underlying cause of which is atherosclerosis, and how the analytical technique of nuclear magnetic resonance (NMR) spectroscopy is being utilised to gain a better understanding in this area.

In the developed world atherosclerosis is the number one cause of death [1], and it is projected that this will be true globally in the near future [2]. As well as major morbidity and mortality implications, the financial burden that this condition presents is huge with the cost estimated at over $\$ 500$ billion in the United States alone in 2010 [3]. Heart attack and stroke are the main manifestations of this inflammatory disease. Immune responses play an important role in complex events, including accumulation of lipids, that eventually result in plaque formation [4]. A greater understanding of disease initiation and development could reduce the effects of this worldwide problem, and NMR spectroscopy has the potential to contribute to this cause.

Nuclear magnetic resonance spectroscopy is a widely used technique in chemistry that provides detailed information on the structure of molecules and their relative concentrations in complex mixtures such as biological fluids or extracts from cells or tissues [5-10]. Detailed protocols for the preparation and analysis of common fluids and tissue for such studies are available [11-13]. NMR spectroscopy may also be conducted on solid state samples, complementing magnetic resonance imaging (MRI) studies. While MRI can undoubtedly be informative in monitoring the progression of disease states, its practice and data interpretation procedures are significantly different to those used with solution phase systems; thus, work in this area is not included in the current paper. NMR spectroscopy may be used to monitor specific components of complex matrices; these components are categorised as either high (generally lipid or lipoprotein containing) or low molecular weight entities (such as amino acids); this division is utilised for the purpose of this paper.

The output of an NMR experiment is a spectrum. The signals in the spectrum arise from nuclei in molecules which possess a property called "spin." Only certain nuclei have "spin"; ${ }^{1} \mathrm{H},{ }^{13} \mathrm{C}$, and ${ }^{31} \mathrm{P}$ are the most important in the context of biologically relevant molecules. The $x$-axis of the spectrum is the chemical shift scale; the position of the spectral lines depends on the chemical environment of the nucleus, and is measured in parts per million (ppm). The area under each 


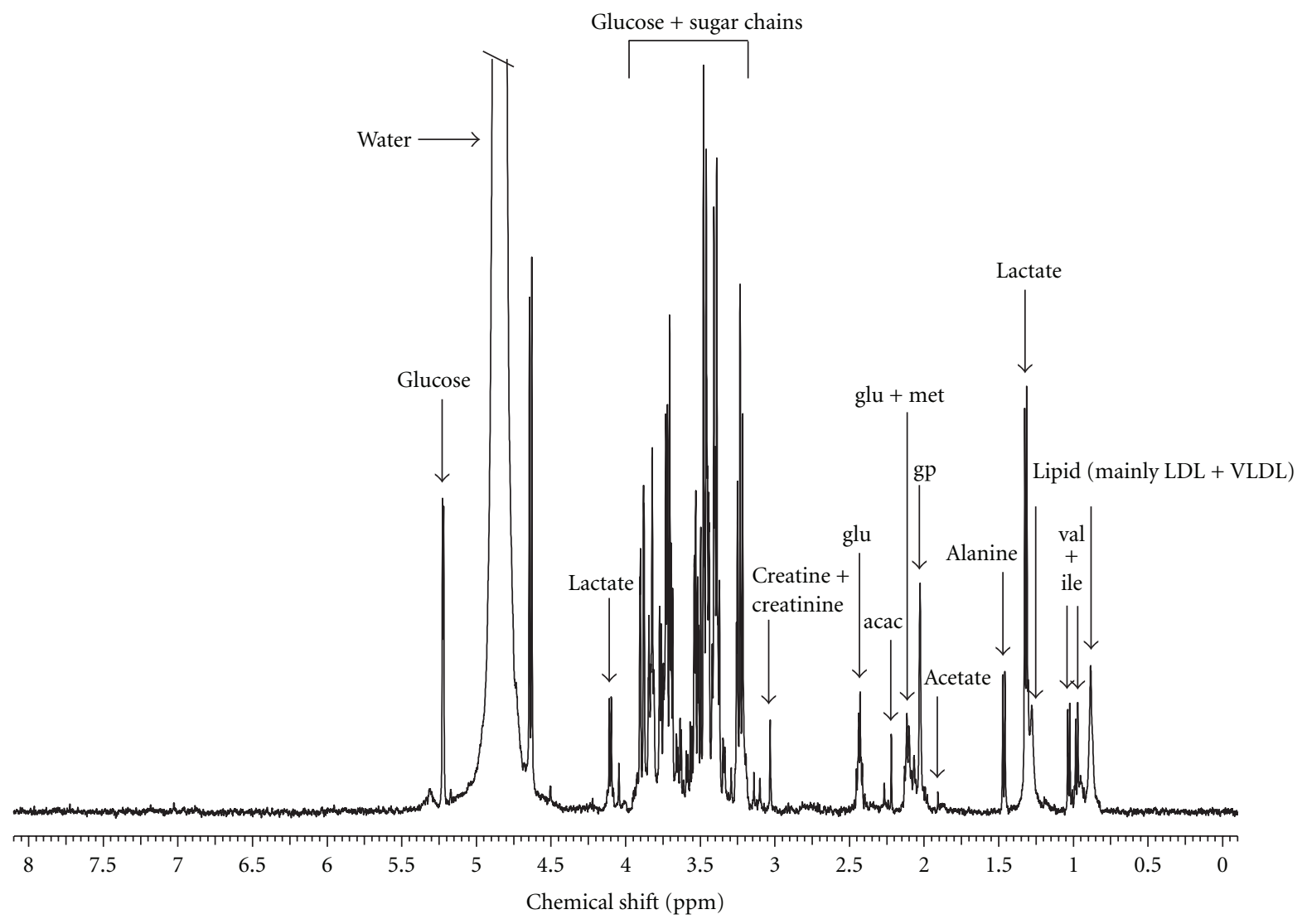

Figure 1: ${ }^{1} \mathrm{H}-\mathrm{NMR}$ spectrum of human blood plasma using CPMG (Carr-Purcell-Meiboom-Gill) pulse sequence to attenuate the majority of the signals from high molecular weight specifies such as albumin and some lipid molecules. Acac: acetoacetate; glu: glutamate; gp: glycoprotein; ile: isoleucine; LDL: low density lipoprotein; met: methionine; val: valine; VLDL: very low density lipoprotein.

signal is directly related to the concentration of the nucleus and this is key to the utility of the technique. A variety of NMR experiments may be conducted to effectively filter the information content of the spectrum, that is signals from high or low molecular weight (see above) entities may be selectively detected without the need for physical separation of these species. An illustration of an NMR spectrum is provided in Figure 1.

The way in which the spectral data is interrogated depends on whether a "targeted" or "global" search is being conducted. If targeted, then the shape of, or area under, the resonances for specific molecules is quantified. In the global (so-called nonhypothesis based) search the whole of the spectrum is considered and compared across, for example, different patient cohorts. As the global approach is generally concerned with identifying and quantifying low molecular weight components (i.e., metabolites), these are called metabolomics or metabonomics studies, and the data collected is interrogated using a chemometric approach [14].

There are a number of reviews which describe the theoretical background to the NMR technique $[15,16]$ and a chemometric approach to data analysis $[17,18]$, as used in metabolomics. Such detail is therefore not reproduced here, rather a brief overview is given below.
Following acquisition of the NMR response and processing to produce well-phased and baseline-corrected spectra, the first stage of chemometric analysis traditionally involves data reduction. This is usually achieved through "binning," whereby the spectrum is divided into smaller regions (bins), and the area under the signals contained in each bin is obtained. This process is performed on each spectrum, from all patients for example. The reduced data, a two-dimensional matrix of bin numbers (the number of columns being the number of bins which may be several 100, which are directly related to chemical shift), and number of spectra (the number of rows being the number of patients sampled, for example) are then normalised to accommodate concentration changes which are patient-dependent and scaled to reduce the dominating effect which high concentration components of the biofluid can have on the subsequent statistical analysis. The data are then interrogated using a multivariate statistical analysis (MVA) approach. Principal components analysis (PCA) is one such MVA method, which reduces the number of bins into a smaller number of variables called principal components (PCs) that describe the majority of the variation in the spectral data. This analysis produces a scores plot and a loadings plot which can be used, for example, to identify trends across a set of patients 


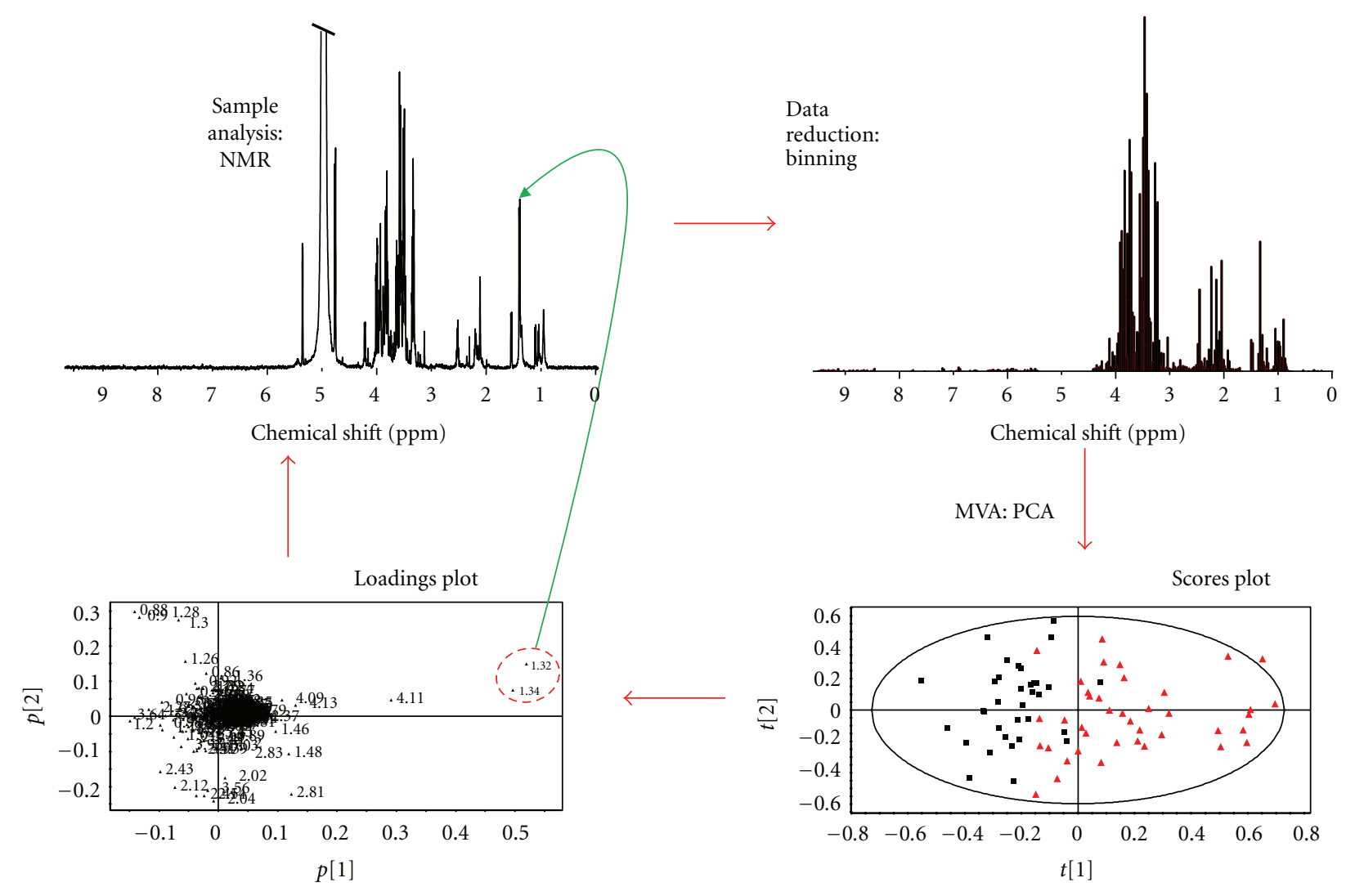

FIGURE 2: A typical metabolomics procedure requires data acquisition using a suitable technique, data reduction and finally multivariate analysis (MVA) to interrogate the sample data. For this example, human blood plasma is analysed using NMR spectroscopy followed by binning as the data reduction method. Principal components analysis (PCA) is the MVA employed producing a scores plot and a loadings plot which can be used to identify trends across the set of patients.

(Figure 2). Partial least-squares discriminant analysis (PLSDA) is another MVA method, but a quantitative relationship is determined between the aforementioned two-dimensional matrix and another matrix, such as gender or some other category [18].

Amongst the review articles covering NMR-based metabolomics applications, some include subsections relevant to cardiovascular studies [19-21], and there are more general reviews concerning both clinical and nonclinical applications of NMR [22, 23]. In contrast, this paper will focus on key publications in which NMR spectroscopy has been employed, where the aforementioned general disease state, or its implications, has been the main point of interest.

\section{Methods}

A systematic review was conducted of articles in the Embase and Web of Knowledge (WOK) databases. For both databases, the same keywords were used, with NMR always being one of the search terms (Table 1). In the absence of a time period restriction, almost 2000 articles were sourced. As there have been considerable advances in both instrumentation and data handling software in recent years, the time frame was reduced and restricted to January 2000 to June 2012. This still resulted in approaching 1000 articles.
TABLE 1: Search terms used for the compilation of literature for this paper.

\begin{tabular}{l}
\hline Keyword: NMR \\
\hline Paired terms: \\
arter* (artery, arteries, arterial, arteriosclerosis, arteriosclerotic) \\
ather* (atherosclerosis, atherosclerotic, atheroma, atherogenesis, \\
atherogenic) \\
peripheral arterial disease \\
claudic* (claudication, claudicant) \\
critical isch*emi* (ischemia, ischaemia, ischemic, ischaemic)
\end{tabular}

The keyword was paired with each of the five terms in the search engines used, for example "NMR AND arter.*” Terms within brackets are examples of truncation and alternative spellings that are encompassed by the wildcard feature.*

Clearly due to the broad nature of the disease and versatility of NMR, it is not possible to produce a review that is both substantive and general including all the search results. Consequently the abstracts for these were searched and the number of reports considered in detail here reduced to around 20 on the basis of the novelty of the work, the depth of the analysis reported, and the direct relevance to the disease area. In this way, around half of the articles 


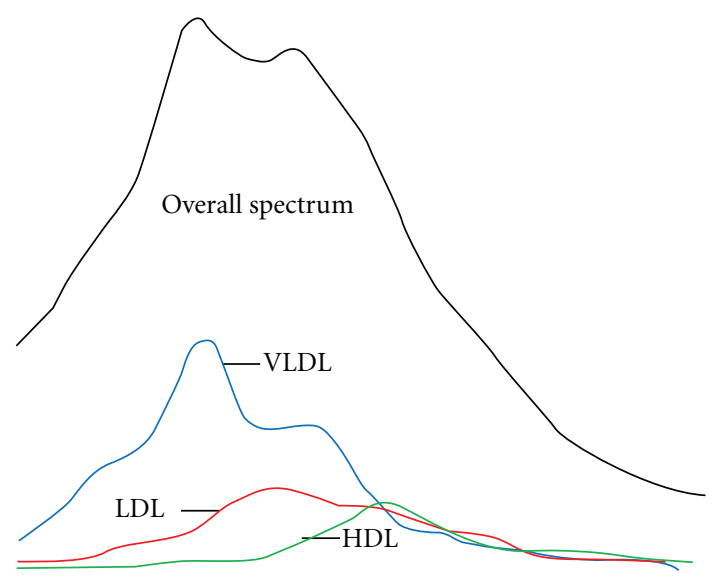

FIGURE 3: Simulated spectra of lipoprotein components, including very low density (VLDL), low density (LDL), and high density lipoproteins (HDL), that contribute to the simulated methyl region (around $0.9 \mathrm{ppm}$ ) of a spectrum.

considered relate to the measurement of lipid and lipoprotein content, the other half utilising the metabolomics approach. For completion, a small number of articles relating to other potential indicators are also included.

\section{Results}

3.1. Measurement of Lipids and Lipoproteins. Current prevention guidelines recommend measurement of standard lipids, including quantification of cholesterol or triglyceride content of lipoproteins, to assess the risk of cardiovascular disease (CVD), but it has been suggested that alternative lipoprotein measures, including lipoprotein subclass profiling of plasma or serum, using NMR spectroscopy may improve risk prediction [24]. This particular NMR application aims to provide information in a much shorter time period than the established ultracentrifugation method; results can be gained after a few minutes rather than many hours. Additionally, no sample pretreatment or physical or chemical decomposition is required [25], nor fasting of patients before sample donation [26]. There are two leading groups working in this area headed by J.D. Otvos and M. Ala-Korpela. Indeed, Otvos has established a lipoprotein profiling company, currently named LipoScience [27]. The data handling adopted by Otvos and coworkers as part of their analysis platform has, however, been criticised by Ala-Korpela and coworkers with regards to its accuracy in resolving the lipoprotein signal into individual component signals [28]; hence, they have used alternative methodologies including metabolomics [29].

Lipoprotein particles have different diameters and are generally categorised in terms of diameter range; each category or subclass givs rise to representative signals in the NMR spectrum. However, these signals are broad and the chemical shift dispersion is not great (Figure 3).

The basis for the analysis is described in brief here with references given as appropriate for a more detailed treatment. In ${ }^{1} \mathrm{H}$ NMR spectra of plasma, lipid methyl $\left(\mathrm{CH}_{3}\right)$ groups are observed at $\sim 0.8 \mathrm{ppm}$, whilst methylene $\left(\mathrm{CH}_{2}\right)$ groups appear at $\sim 1.2 \mathrm{ppm}$; larger lipids have the greatest downfield shift (highest ppm value), and smaller lipids have the greatest upfield shift in each spectral region. Otvos et al. concentrate on analysis of the methyl signals, whilst Ala-Korpela et al. focus on the methylene region. The methyl and methylene groups are contained both in the core (cholesterol ester and triglycerides) and in the surface shell (phospholipid and unesterified cholesterol) of the lipoprotein. To a close approximation, the number of methyl groups contained within a particle depends only on the diameter of the particle [30]. From the methyl group signal, the concentration of the subclass can be determined. Using purified standards information the concentration of subclasses can be calculated, and, hence, also that of lipoprotein classes as well as HDL (high density lipoprotein) and LDL (low density lipoprotein) cholesterol and triglyceride concentrations. There is a good correlation between NMR spectroscopy-derived and chemically measured lipid levels $[28,30]$. A number of studies employing the lipid/lipoprotein analysis approach are considered below.

As a consequence of mutations in the LDL receptor gene, levels of low-density lipoprotein cholesterol (LDL-C) are elevated, from birth, in children with familial hypercholesterolemia (FH) [31]. These children are predisposed to CVD and were shown to have a more highly atherogenic lipid profile than non-FH siblings. Pravastatin had previously been shown to safely reduce LDL-C levels in children with FH. In this study the drug was found to alter the concentrations of the various lipid types [31] (Table 2) in such a manner to indicate that pravastatin therapy cannot fully restore, but can improve, the lipoprotein profile of $\mathrm{FH}$ children to one that is normal. However, the significance of small and large LDL levels has been questioned by Mora et al. [32].

Levels of HDL were the focus of investigation in patients with stable coronary arterial disease (CAD) taking statins and receiving either placebo or a form of $\alpha$-tocopherol (AT) for a two year period [33]. AT therapy did not affect total, small, medium or large HDL particle concentrations compared with baseline or placebo, nor the equivalent categories for LDL and VLDL (very low density lipoprotein) concentrations. This led to the conclusion that AT does not cause the attenuation of statin therapy, as observed in a previous study using the same form of AT combined with a mixture of vitamins [34].

Although prolonged survival has increased amongst individuals with human immunodeficiency virus (HIV) infection, in part due to antiretroviral therapy (ART), CVD is now a leading cause of death and the disease rate is higher for individuals with HIV than without [35]. HDL levels were lower for patients at the study start who subsequently had a CVD event, prior to the CVD event independent of initial levels and who received intermittent ART compared to continuous treatment.

Differences in the sizes of lipoprotein particles and concentrations of lipoprotein subclasses between males and females were investigated to determine whether gender was a risk factor for coronary heart disease (CHD) [36]. Compared to women, men had smaller LDL and HDL particles but 
larger VLDL particles. Samples were further categorised according to age (less than 45 years old, 45 to 54 , and 55 or greater), and it was reported that the difference in HDL particle size between men and women decreased by $\sim 50 \%$ with age as does the difference in CHD risk. Little change in LDL particle size with age was observed, but the difference in total LDL particles between sexes narrowed with age: the over 55 category having similar levels whereas at lesser ages, women have much lower levels than men. The increased LDLcholesterol concentration of older women was attributed to higher concentrations of intermediate and large LDL rather than small LDL. After adjustment for lipid and lipoprotein concentrations, the sex differences in the mean sizes of lipoprotein particles persisted indicating that women have a less atherogenic subclass profile than men [36].

In a study of atherosclerosis in which small LDL levels were classified into 4 categories and large LDL levels classified into 4 categories per small LDL group, a positive correlation was found between intima-medial thickness (IMT) and LDL concentration within each of the small LDL categories [32]. Additionally, a significant linear association of large LDL concentration with IMT was revealed along with a greater difference in IMT per large LDL particle compared with small LDL. It was concluded that a strong confounding factor of the association of large LDL with subclinical atherosclerosis was small LDL and could explain why it is often thought that larger LDL size is less atherogenic.

Increased LDL levels in the Alaskan Eskimo population, which has a high cardiovascular risk but little hyperlipidemia, were linked to factors associated with atherosclerosis [37]. A greater carotid IMT was associated with total LDL particle concentration independently of other traditional risk factors. Carotid plaque was associated with greater concentrations of large LDL particles and a reduction in the number of large VLDL particles and VLDL size. Additionally, large LDL particle concentrations were significantly, and inversely, associated with VLDL size. Small VLDL particles can enter the subendothelial space more easily resulting in the suggestion that VLDL could have an atherothrombotic role which would lead to plaque formation. Large LDL particles are implicated in CVD in agreement with Mora et al. [32] and not just small ones as concluded by some previous studies [38].

Metabolic syndrome (Met Syn) in both men and women has been studied in relation to lipid and lipoprotein profiling. As the number of components of Met Syn increased, LDL particle numbers increased, especially for small LDL. However, a higher small LDL particle number was not associated with an increased CVD event rate [39]. Two suggestions were made regarding small LDL: it does not directly promote atherosclerosis and instead acts as a marker or it might predict CVD risk when considering all patients rather than just Met Syn individuals. Changes in small LDL and large VLDL levels mirrored those of primate studies [40].

Diabetes is known to be a confounding factor in CVD; hence, diabetics are an interesting group to study with regard to lipid metabolism. Considering a cohort of nondiabetic and, at study end, diabetic patients, VLDL size and small HDL levels were found to differ significantly [41]. At the study start, the latter had a lipoprotein profile more similar to those who initially had diabetes than the former indicating a proatherogenic profile in prediabetic individuals.

As well as identifying many significant differences in lipoprotein subclass concentrations and size in women, it has been shown that LDL particle concentration performed well for CVD risk prediction, and the measurement was equal to but not better than the total/high-density lipoprotein cholesterol ratio that is readily obtained. This supports the current recommendation that standard lipids, in particular the total/high-density lipoprotein cholesterol ratio, can be used for routine cardiovascular disease risk assessment [42], though NMR spectroscopy provides useful additional information.

Lipoprotein profiles have been investigated both in human and nonhuman studies as well as the effects of drugs and treatments including, but not limited to, statins. Table 2 contains a summary of the articles discussed. A wealth of information regarding VLDL, LDL, and HDL levels, in terms of average size and small, medium, large, and total concentrations, has complemented and often furthered knowledge that is available from standard biochemical testing regarding cardiovascular events. Despite a study showing no increased advantage of CVD risk prediction versus biochemically derived measures [42], given the extra information obtainable there are lots of atherosclerotic disease-related study areas that could potentially benefit from NMR spectroscopy-based lipoprotein profiling. This is supported by the American Association for Clinical Chemistry Lipoprotein and Vascular Diseases Division Working Group's recommendation to use LDL particle number concentration for assessing and monitoring patients at risk for CVD rather than LDL-C [24]. Confounding factors have to be carefully considered and many studies now adjust the significance of findings based on different confounding factors which can reduce the number of changing lipoproteins.

3.2. Metabolomics. One of the first human studies using metabolomics concluded that diagnosis of coronary heart disease was possible because over $90 \%$ of patients with stenosis of all three major coronary vessels were able to be distinguished from patients with angiographically normal coronary arteries, with a specificity of greater than 90\% [43]. However, this finding was contested by Kirschenlor et al. [44]. Brindle et al. [43] included mostly males (34 from 36) in the triple vessel disease (TVD) group but mostly females (23 from 30 ) in the normal coronary artery (NCA) group. Nearly all of those with CAD were taking statins, which decrease cholesterol, LDL, and triglyceride levels. These two confounding factors, gender and drug treatment, were proposed as the reason for the nonreproducibility of sensitivity and specificity levels [44]. Additionally, the severity of cardiovascular disease, as defined by stenosis of 1,2 , or 3 of the major coronary arteries, was analysed and clear separation between groups was visualised even though all patients were males. Kirschenlor et al. [44] showed that 100\% of samples were correctly predicted, based on gender, and all at a confidence limit of greater than $99 \%$. Higher prediction percentages were observed for either males or females taking 


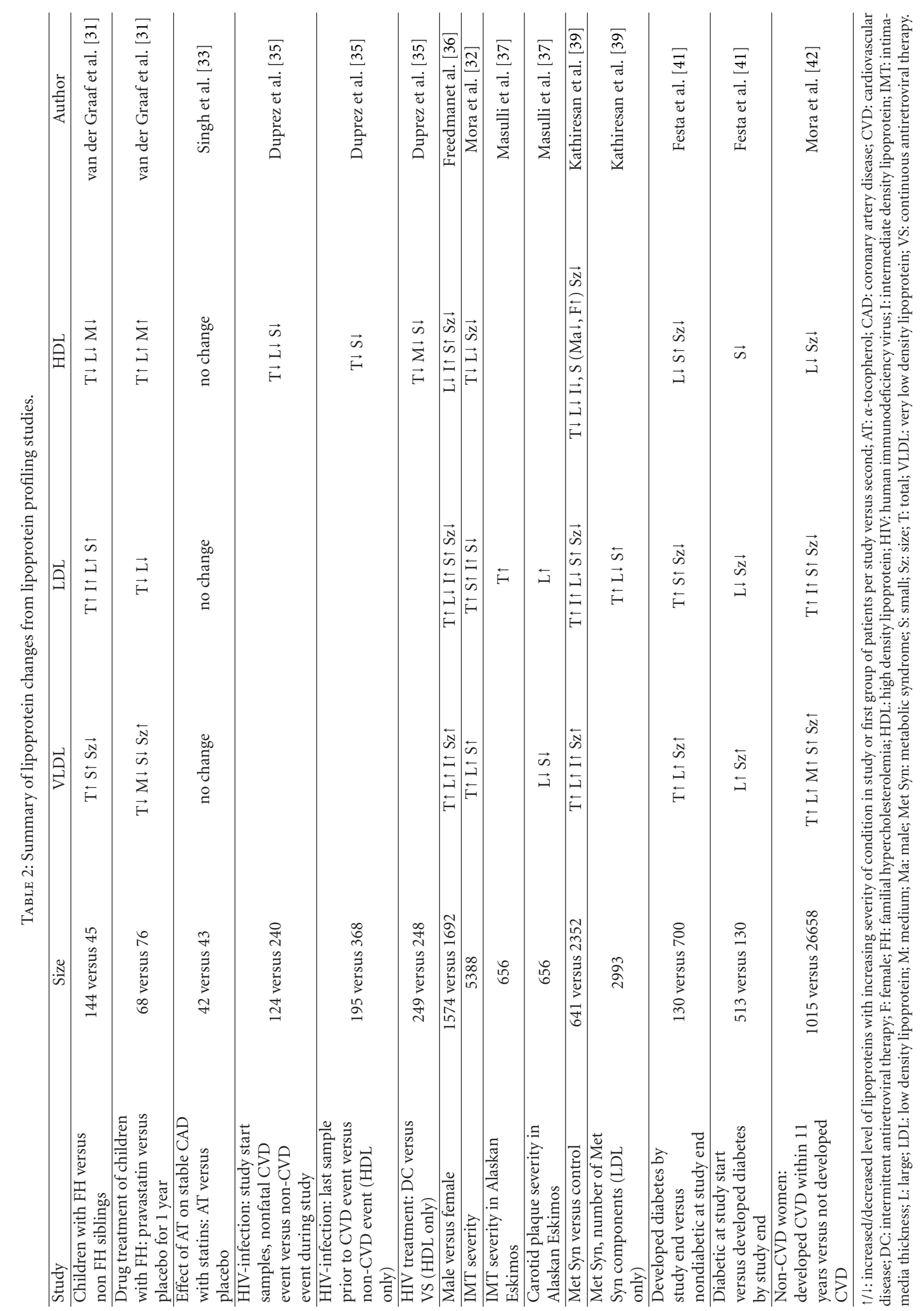


statins than same sex untreated individuals, although the percentage was lower than that based on gender. Together, this is indicative of gender being a strong confounding factor and statin treatment a weaker, but still important, confounding factor. As a result, females were excluded, and statin and nonstatin groups were analysed separately when predicting CAD and non-CAD groups thus providing $80.3 \%$ and $61.3 \%$ correct predictions, respectively. NMR spectroscopy was a weak predictor of CAD compared with angiography but provided clear identification of samples based on other factors [44].

In a study conducted by Bernini et al. a large number of plasma samples from healthy donors were classified, where data existed, into quintiles based on LDL, HDL, triglycerides, total cholesterol levels, and HDL to LDL ratio. The extreme quintiles' samples revealed a number of metabolites significantly associated with low/high-risk cardiovascular disease profiles [45].

Double cross-validation predicted sensitivity, specificity, and accuracy of extreme quintiles of greater than 95\% with respect to $\mathrm{LDL}, \mathrm{HDL}$, triglycerides, and total cholesterol. When removing all lipid regions, approximately $20 \%$ of the spectrum accuracy with respect to total cholesterol decreased to $75 \%$ but confirmed that more subtle metabolic information than lipid fractions can be identified as distinguishing between samples [45].

Metabolomics and proteomics analyses were performed on human atrial appendage (heart) tissue that was collected from two patient groups and those classified as having atrial fibrillation (AF) abnormal heart rhythm or sinus rhythm (SR) normal heart rhythm [46]. AF patients had severe nonrheumatic valvular disease, whereas SR patients were undergoing either coronary artery bypass grafting (CABG) or valve surgery. Those with preoperative SR either remained SR or developed AF (SR-AF) postoperatively. More than $80 \%$ of patients who were SR pre-CABG surgery and were either SR or SR-AF postoperatively were correctly predicted [46]. In addition to total SR-AF patients having statistically significant lower levels of glucose and creatine than total SR patients, the glucose to acetate ratio was also reduced, as was observed for patients who developed postoperative AF in both CABG and valve surgery sub-classes. The authors also observed a positive correlation between the time of onset of postoperative AF in the CABG group and the ratio of alanine plus lactate (glycolytic end products) to acetate (lipid metabolism end product) indicating that glycolytic inhibition predisposes to AF [46]. Interestingly, due to unchanged glucose levels in persistent AF patients, cardiac accumulation of glycogen has been suggested to occur through impairment of glycogen catabolism. $\beta$-hydroxybutyrate and tyrosine, which form acetoacetate and fumarate during catabolism, were elevated in persistent AF indicating that an important energy source during persistent AF could be ketone bodies.

Teul et al. [47] used NMR spectroscopy and gas-chromatography coupled to mass spectrometry (GC-MS) as complementary techniques for investigation of stable carotid atherosclerosis. Metabolites that showed statistically significant differences between patient and control as identified by NMR spectroscopy are presented in Table 3 . When both techniques were considered, most of the metabolite level changes were characteristic of metabolism alterations due to insulin resistance and hence the Met Syn, despite the majority of patients not being diagnosed as diabetics. Although removal of diabetic patients had little effect on statistical models, 3hydroxybutyrate (3-HB) and glycerol became significantly increased in patients versus controls, whereas, previously, both had been increased but not significantly. Increases in 3$\mathrm{HB}$ and acetoacetate have been suggested to increase in insulin signalling deficiency cases by increased acetyl co-enzyme A (AcCoA) from lipolysis and $\beta$-oxidation of fatty acids. Increased lipolysis could be the source of increased glycerol in patients. Amino acids will less likely be used for protein synthesis due to lack of insulin response and a decrease in amino acid availability due to disposal as an energy source has been forwarded to explain reduction in alanine and proline levels. Creatinine is strongly associated with amino acid utilisation. 3-HB levels were correlated with those of many other metabolites including strongly positive with creatinine and acetoacetate, positively with proline, and strongly negative with alanine.

Prediction of, and metabolites associated with, exerciseinduced ischemia was investigated in serum from patients undergoing exercise single-photon emission computed tomography (SPECT) myocardial perfusion imaging with suspected stable coronary artery disease but without a history of prior myocardial infarction. SPECT identified patients as having or not having exercise-induced ischemia [48]. Lipids at 0.9 and $1.28 \mathrm{ppm}$ were relatively elevated in serum from nonischaemic patients, whereas exercise induced ischaemic patients had elevated levels of lactate, glucose, and longchain amino acids (mainly valine, leucine, and isoleucine). These metabolites contributed to discrimination between the two groups but confounding factors, such as the higher frequency of males among subjects developing ischemia, could explain the discrimination power. Exclusion of the glucose region still resulted in separation between groups allowing the authors to conclude, diabetes did not play a major role in the study.

Mäkinen et al. [29] have presented complex metabolomics analyses of serum via multifactorial statistical methods of diabetic nephropathy and T1DM (type 1 diabetes mellitus). Diabetic nephropathy is the most severe complication of T1DM patients and is associated with a high risk of atherosclerotic diseases and premature death. The urinary albumin excretion rate is the gold standard used to determine diabetic nephropathy, but multiple overnight or 24 hour urine samples are required. Metabolomics was performed to determine whether the technique could be of diagnostic value.

Associations between a number of biochemical variables, such as 24 hour urine albumin and serum HDL2 cholesterol levels, and spectral intensities were observed. A negative association was present between glucose and biochemically measured serum sodium. Regression models were used for nephropathy diagnosis for both NMR spectral features and biochemical measurements. Both models clearly separated T1DM and non-T1DM samples. Additionally, T1DM samples were classified correctly to a similar extent by a number 


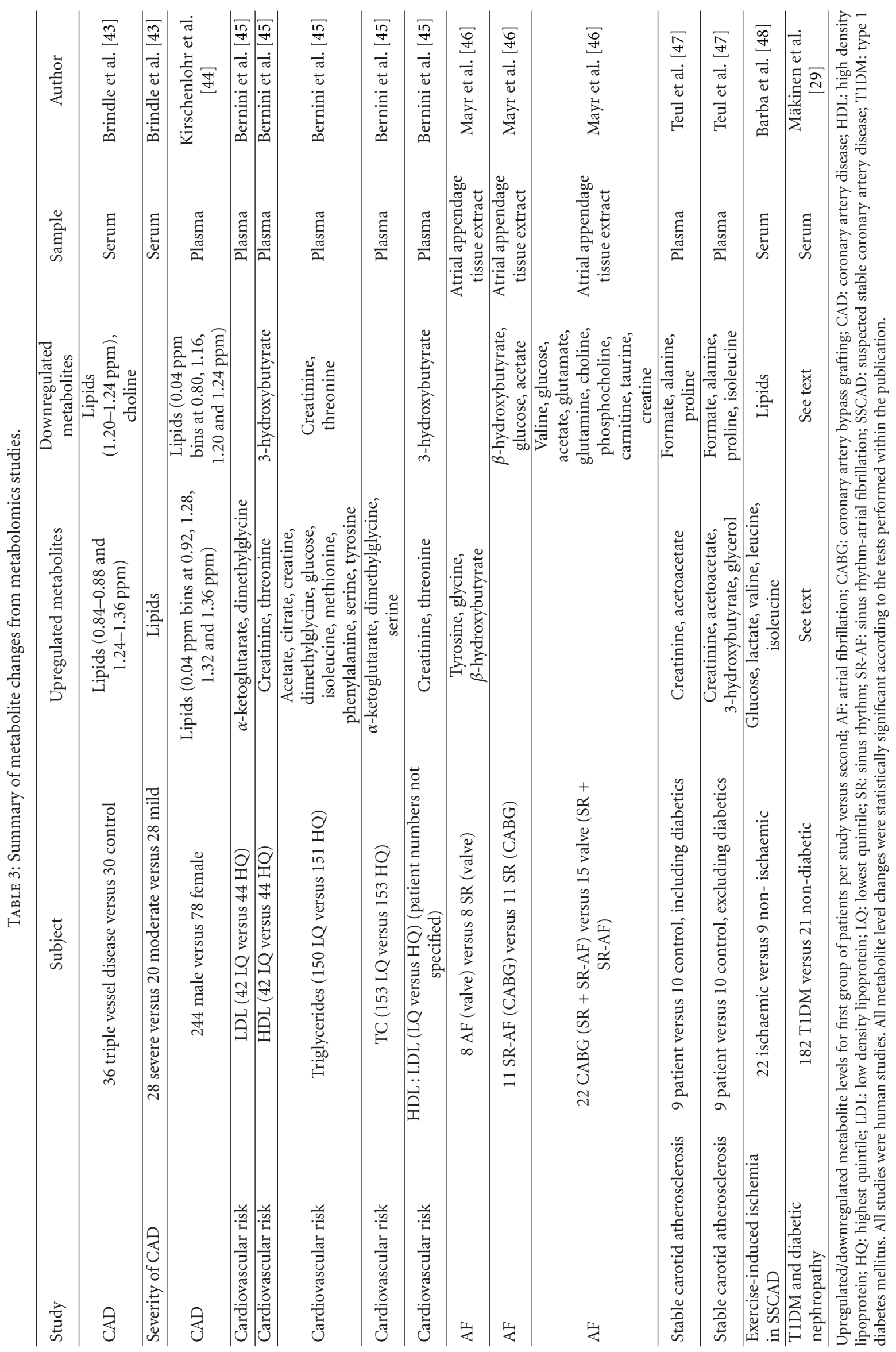


of measures using either quantification method (percentages relate to NMR spectroscopy data versus biochemical data). Sensitivity (probability that macroalbuminuria was detected for patients who had diabetic nephropathy) of 87.1 versus $83.9 \%$ and specificity (probability that normoalbuminuria was detected for patients who did not have diabetic nephropathy) of 87.7 versus $95.9 \%$. Predictive value of positive tests (probability that a patient had diabetic nephropathy when the test indicated macroalbuminuria) of 89.0 versus $95.5 \%$ and predictive value of negative tests of 83.6 versus $79.2 \%$. Combined, these data show NMR-based metabolomics distinguished metabolic traits of T1DM and provides comparable diagnostic ability for nephropathy to the gold standard of biochemical variables.

3.3. Other Applications. Chen et al. [49] have provided evidence for the double belt model for the structure of discoidal HDL, being one of the many models that have been derived such as picket fence and belt models, including double, hairpin double and flexible registry double belt submodels, but a definitive answer has not been elucidated.

Discoidal HDL is a disk-shaped intermediate of mature HDL that activates the enzyme lecithin cholesterol acyltransferase (LCAT), which permits cholesterol ester addition to HDL particles. Discoidal HDL contains two apolipoprotein AI (ApoAI) molecules, the major protein component of HDL, but usually a small percentage is the lipid-poor form of ApoAI rendering it unsuitable for NMR spectroscopy analysis due to the requirements of high homogeneity and purity not being met. Ultracentrifuged HDL, which did not contain the lipid-poor form of ApoAI, was purified into small and large reconstituted HDL (rHDL) for NMR spectroscopy analysis. Two-dimensional (2D) NMR spectra (which provide better spectral or chemical shift resolution) of isotope $\left({ }^{15} \mathrm{~N}\right)$ enriched protein were used with two different sizes of rHDL and indicated different tertiary structures for ApoAI. The NMR data was consistent with one form of ApoAI molecules being present hence indicating symmetry in the complex. Therefore, the double belts of the $\alpha$-helix structure of ApoAI should be identical whether it forms an intermolecular or an intramolecular bundle.

Epand et al. [50] have demonstrated through various ${ }^{13} \mathrm{C}$ cross-polarisation/magic angle spinning (CP/MAS) NMR spectroscopy experiments the difference in cholesterol crystallite formation in lipid rafts, although the molecular reasoning for the difference could not be rationalised by NMR spectroscopy.

Lipid rafts are membranes that are composed of phospholipids with an enrichment of cholesterol and sphingolipids compared to nonraft membrane areas and have a higher degree of order [50]. Cholesterol crystallites form at higher cholesterol concentrations, but this level depends on the phospholipid; a lesser concentration is needed in phosphatidylserine bilayers than in those of phosphatidylcholine. Comparison by NMR spectroscopy of mixtures of cholesterol with 1-palmitoyl-2-oleoylphosphatidylserine (POPS) and with 1-palmitoyl-2-oleoylphosphatidylcholine (POPC) was performed, both above and below the concentration required for formation of cholesterol crystals [50].
High body mercury content has been associated with atherosclerosis, so the question of whether mercury can promote lipid peroxidation has been addressed [51]. ${ }^{1} \mathrm{H}$ spectra of LDL extracts showed that the olefinic double bond (5.3 ppm), arachidonic acid (2.79 ppm), and linoleic acid (2.75 ppm) signals decreased slightly, whilst the double bond signal for linoleic increased slightly with mercury levels. Both indicate that mercury weakly promotes nonenzymatic peroxidation. However, mercury levels used were $10 \mu \mathrm{mol} / \mathrm{L}$ which is much higher than in whole blood $0.01-0.1 \mu \mathrm{mol} / \mathrm{L}$, and, given the small changes in signal areas under realistic physiological concentrations, mercury has next to no effect on the direct nonenzymatic peroxidation of LDL, whereas copper heavily promotes this method.

Ibuprofen has been suggested to inhibit LDL oxidation, so the interaction between ibuprofen and phospholipids in lipoprotein particles in human plasma was investigated [52]. Various concentrations of ibuprofen, but all above the saturation concentration for albumin binding, revealed chemical shift changes for signals corresponding to $-\mathrm{N}^{+}\left(\mathrm{CH}_{3}\right)_{3}$ headgroups of phosphatidylcholine (PC) and sphingomyelin (SM). Larger shift changes occurred as the ibuprofen concentration increased, and the SM headgroup signal changed more than that of the PC equivalent. NMR-based diffusion measurements [53] were also employed to suppress signals from low molecular weight metabolites and to differentiate between particles on the basis of molecular weight. Consideration of chemical shift and diffusion information led to the conclusion that interaction of ibuprofen with phospholipids, or ibuprofen binding inducing structural changes in the lipoproteins, is possible and might explain the mechanism by which this drug protects against oxidative modification of lipoproteins.

\section{Conclusion}

The inherent accuracy and reproducibility of NMR spectroscopy allow subtle differences in lipoprotein and metabolic profiles to be detected. Analysis of such data can provide information about changes that are underlying due to the population, for example, sex, and age, or related to initiation and development of cardiovascular disease. As more information is obtained and applications of NMR spectroscopy in cardiovascular disease increase, there is potential that a route towards reducing the burden of this worldwide problem may become evident.

\section{Conflict of Interests}

There is no financial arrangement or other relationship that could be construed as a conflict of interests.

\section{Authors' Contribution}

W. Yabsley conducted the research for this paper and wrote various drafts. S. Homer-Vanniasinkam identified the need for work in this area. J. Fisher supervised W. Yabsley 
in his research and edited the paper's drafts. S. HomerVanniasinkam and J. Fisher commented on the final draft of the paper.

\section{Acknowledgment}

W. Yabsley received White Rose Ph.D. studentship fund from the Engineering and Physical Sciences Research Council (EPSRC).

\section{References}

[1] R. Stocker and J. F. Keaney, "Role of oxidative modifications in atherosclerosis," Physiological Reviews, vol. 84, no. 4, pp. 1381$1478,2004$.

[2] G. K. Hansson and P. Libby, "The immune response in atherosclerosis: a double-edged sword," Nature Reviews Immunology, vol. 6, no. 7, pp. 508-519, 2006.

[3] D. Lloyd-Jones, R. J. Adams, T. M. Brown et al., "Heart disease and stroke statistics-2010 update: a report from the American heart association," Circulation, vol. 121, no. 7, pp. e46-e215, 2010.

[4] A. M. Lundberg and G. K. Hansson, "Innate immune signals in atherosclerosis," Clinical Immunology, vol. 134, no. 1, pp. $5-24,2010$.

[5] N. E. Baskind, C. McRae, V. Sharma, and J. Fisher, "Understanding subfertility at a molecular level in the female through the application of nuclear magnetic resonance (NMR) spectroscopy," Human Reproduction Update, vol. 17, no. 2, pp. 228 241, 2011.

[6] E. Turner, J. A. Brewster, N. A. B. Simpson, J. J. Walker, and J. Fisher, "Aromatic amino acid biomarkers of preeclampsiaa nuclear magnetic resonance investigation," Hypertension in Pregnancy, vol. 27, no. 3, pp. 225-235, 2008.

[7] R. P. Hopton, E. Turner, V. J. Burley, P. C. Turner, and J. Fisher, "Urine metabolite analysis as a function of deoxynivalenol exposure: an NMR-based metabolomics investigation," Food Additives and Contaminants A, vol. 27, no. 2, pp. 255-261, 2010.

[8] E. M. Lenz and I. D. Wilson, "Analytical strategies in metabonomics," Journal of Proteome Research, vol. 6, no. 2, pp. 443 458, 2007.

[9] J. C. Lindon and J. K. Nicholson, "Spectroscopic and statistical techniques for information recovery in metabonomics and metabolomics," Annual Review of Analytical Chemistry, vol. 1, no. 1, pp. 45-69, 2008.

[10] J. C. Lindon, E. Holmes, and J. K. Nicholson, "Metabonomics techniques and applications to pharmaceutical research \& development," Pharmaceutical Research, vol. 23, no. 6, pp. 1075-1088, 2006.

[11] O. Beckonert, H. C. Keun, T. M. D. Ebbels et al., "Metabolic profiling, metabolomic and metabonomic procedures for NMR spectroscopy of urine, plasma, serum and tissue extracts," Nature protocols, vol. 2, no. 11, pp. 2692-2703, 2007.

[12] O. Beckonert, M. Coen, H. C. Keun et al., "High-resolution magic-angle-spinning NMR spectroscopy for metabolic profiling of intact tissues," Nature Protocols, vol. 5, no. 6, pp. 10191032, 2010.

[13] C. Y. Lin, H. F. Wu, R. S. Tjeerdema, and M. R. Viant, "Evaluation of metabolite extraction strategies from tissue samples using NMR metabolomics," Metabolomics, vol. 3, no. 1, pp. 55-67, 2007.

[14] J. K. Nicholson, J. C. Lindon, and E. Holmes, “Metabonomics” understanding the metabolic responses of living systems to pathophysiological stimuli via multivariate statistical analysis of biological NMR spectroscopic data," Xenobiotica, vol. 29, no. 11, pp. 1181-1189, 1999.

[15] W. Eisenreich and A. Bacher, "Advances of high-resolution NMR techniques in the structural and metabolic analysis of plant biochemistry," Phytochemistry, vol. 68, no. 22-24, pp. 2799-2815, 2007.

[16] J. H. F. Bothwell and J. L. Griffin, "An introduction to biological nuclear magnetic resonance spectroscopy," Biological Reviews, vol. 86, no. 2, pp. 493-510, 2011.

[17] S. Wold, A. Berglund, and N. Kettaneh, "New and old trends in chemometrics. How to deal with the increasing data volumes in R\&D\&P (research, development and production)-with examples from pharmaceutical research and process modeling," Journal of Chemometrics, vol. 16, no. 8-10, pp. 377-386, 2002.

[18] J. Trygg, E. Holmes, and T. Lundstedt, "Chemometrics in metabonomics," Journal of Proteome Research, vol. 6, no. 2, pp. 469-479, 2007.

[19] S. N. Goonewardena, L. E. Prevette, and A. A. Desai, "Metabolomics and atherosclerosis," Current Atherosclerosis Reports, vol. 12, no. 4, pp. 267-272, 2010.

[20] R. Madsen, T. Lundstedt, and J. Trygg, "Chemometrics in metabolomics - a review in human disease diagnosis," Analytica Chimica Acta, vol. 659, no. 1-2, pp. 23-33, 2010.

[21] C. L. Waterman, C. Kian-Kai, and J. L. Griffin, "Metabolomic strategies to study lipotoxicity in cardiovascular disease," Biochimica et Biophysica Acta, vol. 1801, no. 3, pp. 230-234, 2010.

[22] S. Paasch and E. Brunner, "Trends in solid-state NMR spectroscopy and their relevance for bioanalytics," Analytical and Bioanalytical Chemistry, vol. 398, no. 6, pp. 2351-2362, 2010.

[23] H. Koskela, "Use of NMR techniques for toxic organophosphorus compound profiling," Journal of Chromatography B, vol. 878, no. 17-18, pp. 1365-1381, 2010.

[24] J. D. Brunzell, M. Davidson, C. D. Furberg et al., "Lipoprotein management in patients with cardiometabolic risk. Consensus conference report from the American Diabetes Association and the American College of Cardiology Foundation," Journal of the American College of Cardiology, vol. 51, no. 15, pp. 1512$1524,2008$.

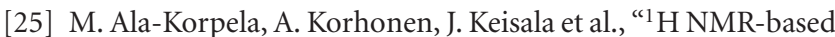
absolute quantitation of human lipoproteins and their lipid contents directly from plasma," Journal of Lipid Research, vol. 35, no. 12, pp. 2292-2304, 1994.

[26] J. D. Otvos, E. J. Jeyarajah, and D. W. Bennett, "Quantification of plasma lipoproteins by proton nuclear magnetic resonance spectroscopy," Clinical Chemistry, vol. 37, no. 3, pp. 377-386, 1991.

[27] LipoScience, "LipoScience A Diagnostic Company," 2010.

[28] M. Ala-Korpela, N. Lankinen, A. Salminen et al., "The inherent accuracy of ${ }^{1} \mathrm{H}$ NMR spectroscopy to quantify plasma lipoproteins is subclass dependent," Atherosclerosis, vol. 190, no. 2, pp. 352-358, 2007.

[29] V. P. Mäkinen, P. Soininen, C. Forsblom et al., "Diagnosing diabetic nephropathy by ${ }^{1} \mathrm{H}$ NMR metabonomics of serum," Magnetic Resonance Materials in Physics, Biology and Medicine, vol. 19, no. 6, pp. 281-296, 2006.

[30] J. D. Otvos, E. J. Jeyarajah, and W. C. Cromwell, "Measurement issues related to lipoprotein heterogeneity," American Journal of Cardiology, vol. 90, no. 8, pp. 22I-29I, 2002.

[31] A. van der Graaf, J. Rodenburg, M. N. Vissers et al., "Atherogenic lipoprotein particle size and concentrations and the 
effect of pravastatin in children with familial hypercholesterolemia," Journal of Pediatrics, vol. 152, no. 6, pp. 873-878, 2008.

[32] S. Mora, M. Szklo, J. D. Otvos et al., "LDL particle subclasses, LDL particle size, and carotid atherosclerosis in the MultiEthnic Study of Atherosclerosis (MESA)," Atherosclerosis, vol. 192, no. 1, pp. 211-217, 2007.

[33] U. Singh, J. Otvos, A. Dasgupta, J. A. de Lemos, S. Devaraj, and I. Jialal, "High-dose $\alpha$-tocopherol therapy does not affect HDL subfractions in patients with coronary artery disease on statin therapy," Clinical Chemistry, vol. 53, no. 3, pp. 525-528, 2007.

[34] B. G. Brown, X. Q. Zhao, A. Chait et al., "Simvastatin and niacin, antioxidant vitamins, or the combination for the prevention of coronary disease," New England Journal of Medicine, vol. 345, no. 22, pp. 1583-1592, 2001.

[35] D. A. Duprez, L. H. Kuller, R. Tracy et al., "Lipoprotein particle subclasses, cardiovascular disease and HIV infection," Atherosclerosis, vol. 207, no. 2, pp. 524-529, 2009.

[36] D. S. Freedman, J. D. Otvos, E. J. Jeyarajah et al., "Sex and age differences in lipoprotein subclasses measured by nuclear magnetic resonance spectroscopy: the framingham study," Clinical Chemistry, vol. 50, no. 7, pp. 1189-1200, 2004.

[37] M. Masulli, L. Patti, G. Riccardi et al., "Relation among lipoprotein subfractions and carotid atherosclerosis in alaskan eskimos (from the GOCADAN Study)," American Journal of Cardiology, vol. 104, no. 11, pp. 1516-1521, 2009.

[38] R. S. Rosenson, J. D. Otvos, and D. S. Freedman, "Relations of lipoprotein subclass levels and low-density lipoprotein size to progression of coronary artery disease in the pravastatin limitation of atherosclerosis in the coronary arteries (PLAC-I) trial," American Journal of Cardiology, vol. 90, no. 2, pp. 8994, 2002.

[39] S. Kathiresan, J. D. Otvos, L. M. Sullivan et al., "Increased small low-density lipoprotein particle number: a prominent feature of the metabolic syndrome in the Framingham Heart Study," Circulation, vol. 113, no. 1, pp. 20-29, 2006.

[40] S. Y. Ding, X. T. Tigno, and B. C. Hansen, "Nuclear magnetic resonance-determined lipoprotein abnormalities in nonhuman primates with the metabolic syndrome and type 2 diabetes mellitus," Metabolism, vol. 56, no. 6, pp. 838-846, 2007.

[41] A. Festa, K. Williams, A. J. G. Hanley et al., "Nuclear magnetic resonance lipoprotein abnormalities in prediabetic subjects in the insulin resistance atherosclerosis study," Circulation, vol. 111, no. 25, pp. 3465-3472, 2005.

[42] S. Mora, J. D. Otvos, N. Rifai, R. S. Rosenson, J. E. Buring, and P. M. Ridker, "Lipoprotein particle profiles by nuclear magnetic resonance compared with standard lipids and apolipoproteins in predicting incident cardiovascular disease in women," Circulation, vol. 119, no. 7, pp. 931-939, 2009.

[43] J. T. Brindle, H. Antti, E. Holmes et al., "Rapid and noninvasive diagnosis of the presence and severity of coronary heart disease using ${ }^{1} \mathrm{H}$-NMR-based metabonomics," Nature Medicine, vol. 8, no. 12, pp. 1439-1444, 2002.

[44] H. L. Kirschenlohr, J. L. Griffin, S. C. Clarke et al., "Proton NMR analysis of plasma is a weak predictor of coronary artery disease," Nature Medicine, vol. 12, no. 6, pp. 705-710, 2006.

[45] P. Bernini, I. Bertini, C. Luchinat, L. Tenori, and A. Tognaccini, "The cardiovascular risk of healthy individuals studied by NMR metabonomics of plasma samples," Journal of Proteome Research, vol. 10, no. 11, pp. 4983-4992, 2011.

[46] M. Mayr, S. Yusuf, G. Weir et al., "Combined metabolomic and proteomic analysis of human atrial fibrillation," Journal of the American College of Cardiology, vol. 51, no. 5, pp. 585-594, 2008.

[47] J. Teul, F. J. Rupérez, A. Garcia et al., "Improving metabolite knowledge in stable atherosclerosis patients by association and correlation of GC-MS and ${ }^{1} \mathrm{H}$ NMR fingerprints," Journal of Proteome Research, vol. 8, no. 12, pp. 5580-5589, 2009.

[48] I. Barba, G. de León, E. Martín et al., "Nuclear magnetic resonance-based metabolomics predicts exercise-induced ischemia in patients with suspected coronary artery disease," Magnetic Resonance in Medicine, vol. 60, no. 1, pp. 27-32, 2008.

[49] B. Chen, X. Ren, T. Neville et al., "Apolipoprotein AI tertiary structures determine stability and phospholipid-binding activity of discoidal high-density lipoprotein particles of different sizes," Protein Science, vol. 18, no. 5, pp. 921-935, 2009.

[50] R. M. Epand, A. D. Bain, B. G. Sayer, D. Bach, and E. Wachtel, "Properties of mixtures of cholesterol with phosphatidylcholine or with phosphatidylserine studied by 13C magic angle spinning nuclear magnetic resonance," Biophysical Journal, vol. 83, no. 4, pp. 2053-2063, 2002.

[51] K. Seppänen, P. Soininen, J. T. Salonen, S. Lötjönen, and R. Laatikainen, "Does mercury promote lipid peroxidation? An in vitro study concerning mercury, copper, and iron in peroxidation of low-density lipoprotein," Biological Trace Element Research, vol. 101, no. 2, pp. 117-132, 2004.

[52] Y. Yang, G. Bai, X. Zhang, C. Ye, and M. Liu, " ${ }^{1} H$ NMR spectroscopic evidence of interaction between ibuprofen and lipoproteins in human blood plasma," Analytical Biochemistry, vol. 324, no. 2, pp. 292-297, 2004.

[53] D. H. Wu, A. D. Chen, and C. S. Johnson, "An improved diffusion-ordered spectroscopy experiment incorporating bipolar-gradient pulses," Journal of Magnetic Resonance A, vol. 115, no. 2, pp. 260-264, 1995. 


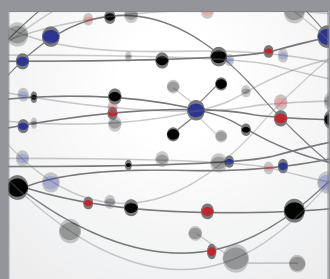

The Scientific World Journal
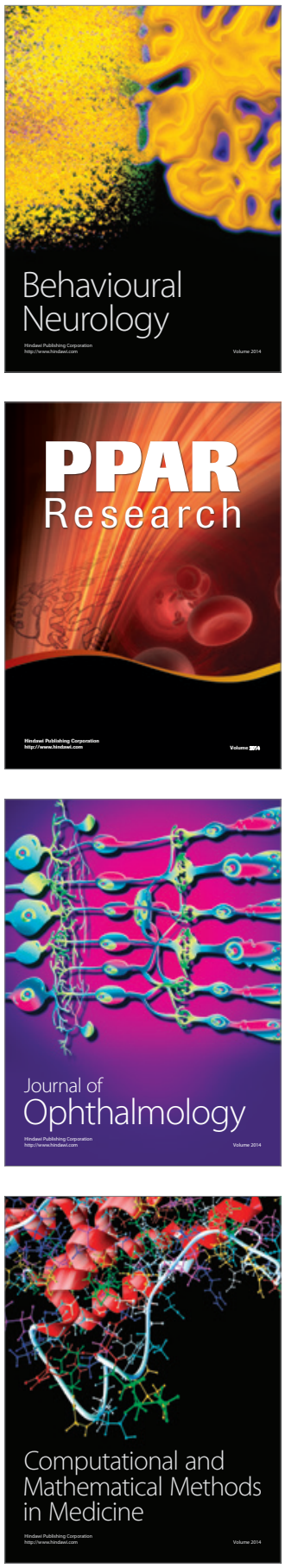

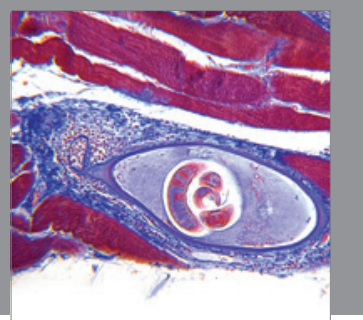

Gastroenterology

Research and Practice
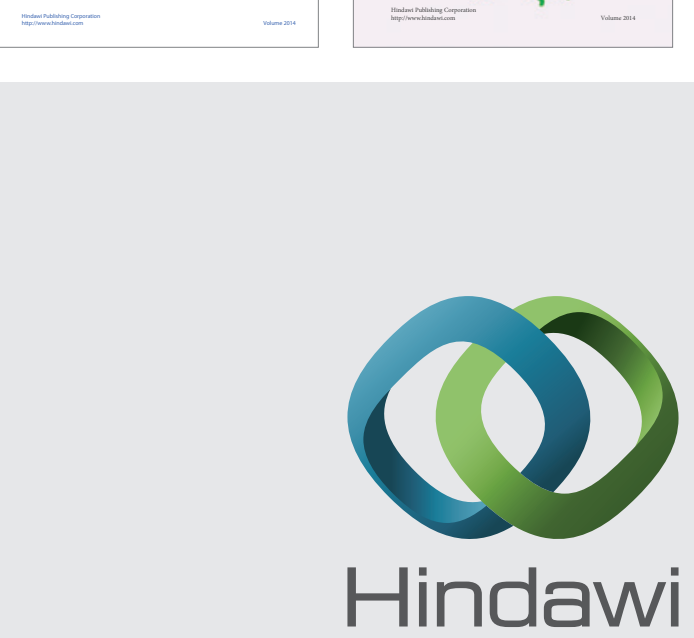

Submit your manuscripts at

http://www.hindawi.com
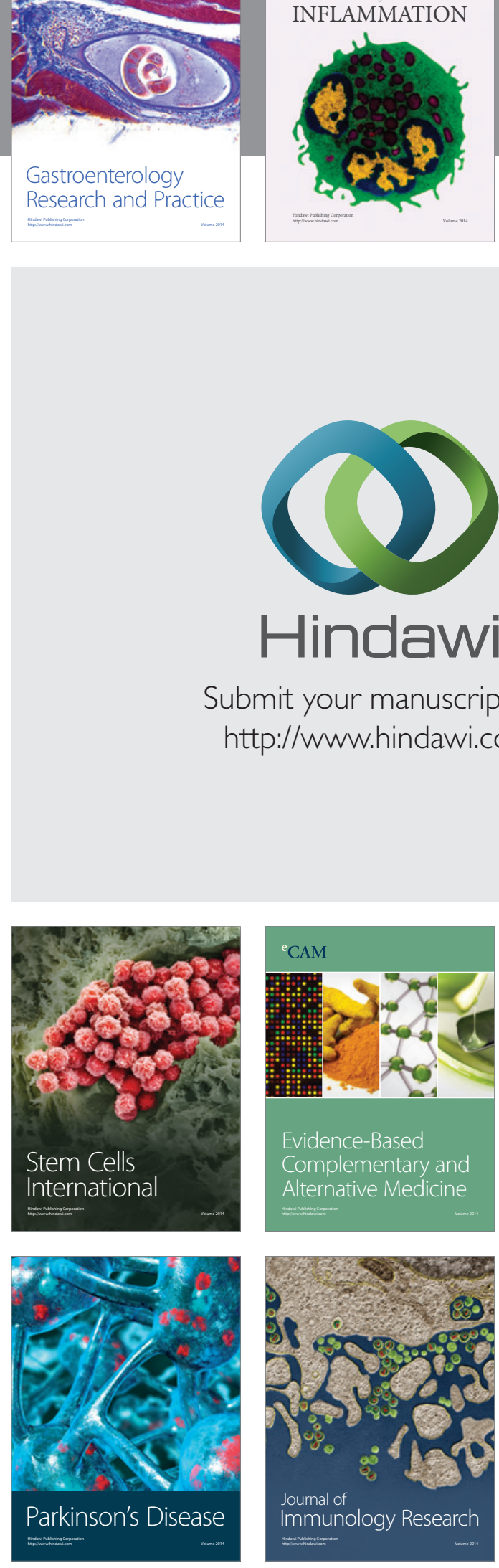

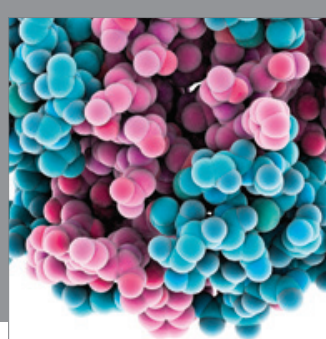

Diabetes Research
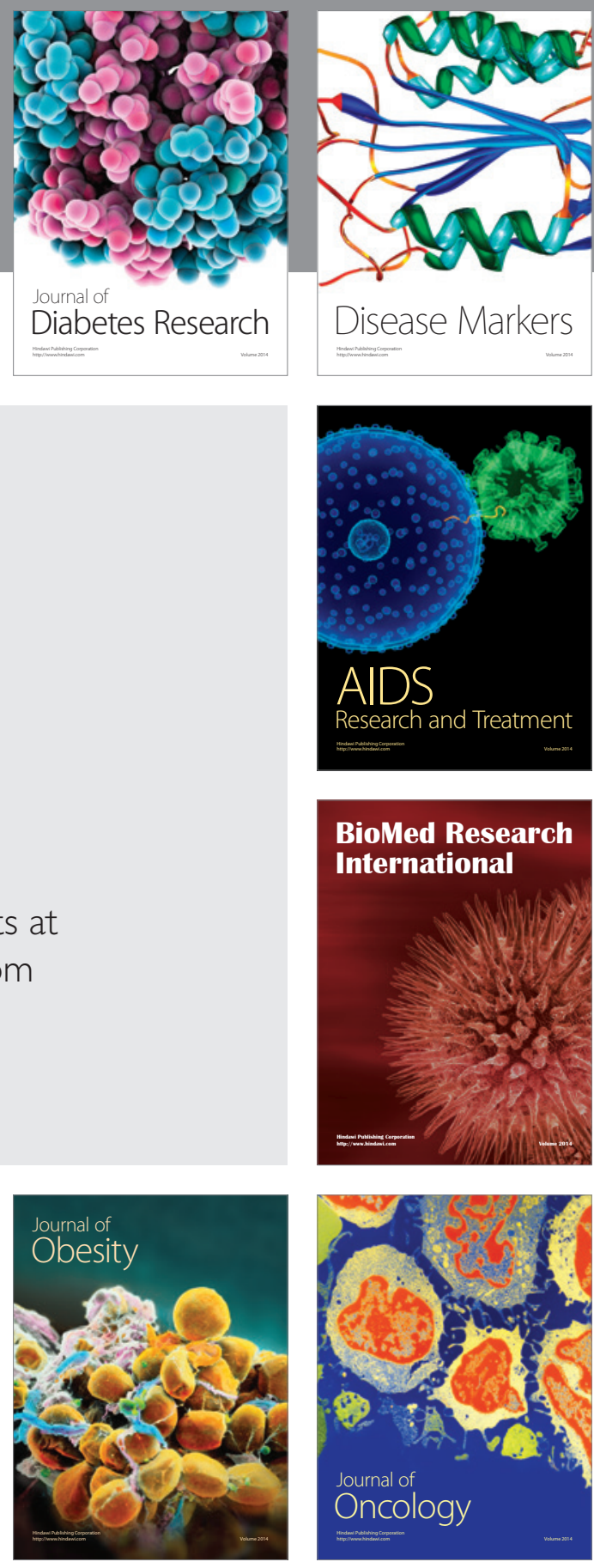

Disease Markers

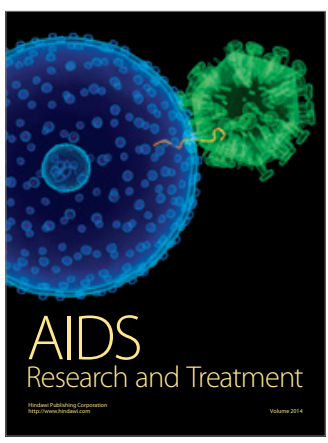

BioMed Research

International
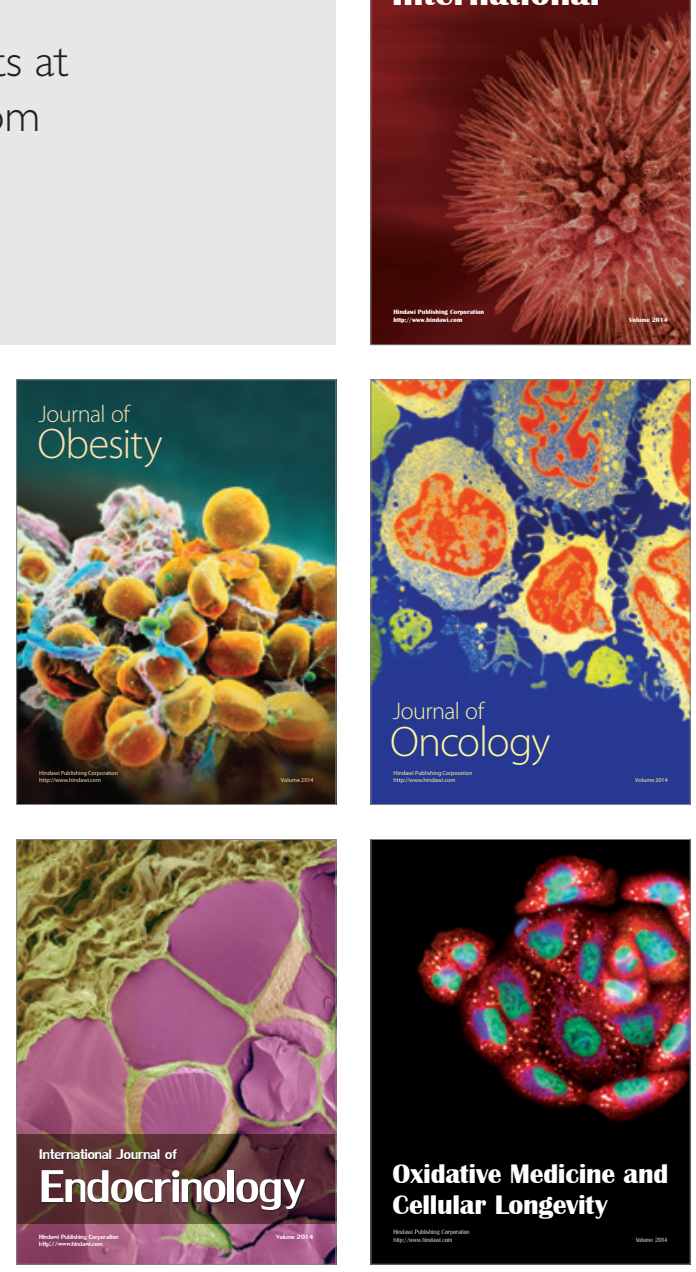